\title{
COVID-19 leukoencephalopathy with subacute magnetic resonance imaging findings of vasculitis and demyelination
}

\author{
Ali Vahedi $^{1} \cdot$ Sean Apap Mangion ${ }^{2} \cdot$ Eli Silber $^{2} \cdot$ Naomi Sibtain $^{1} \cdot$ Julie Chandra $^{1}$
}

Received: 8 February 2021 / Revised: 4 May 2021 / Accepted: 16 May 2021 / Published online: 8 June 2021

(c) Journal of NeuroVirology, Inc. 2021

\begin{abstract}
Severe acute respiratory syndrome coronavirus 2 (SARS-CoV-2) commonly results in a respiratory illness in symptomatic patients; however, those critically ill can develop a leukoencephalopathy. We describe two patients who had novel subacute MRI findings in the context of coronavirus disease 2019 (COVID-19) leukoencephalopathy, which we hypothesize could implicate a potent small-vessel vasculitis, ischemic demyelination and the presence of prolonged ischemia. Recent evidence of the direct neuroinvasiness of SARS-CoV-2 leading to ischemia and vascular damage supports this hypothesis.
\end{abstract}

Keywords COVID-19 $\cdot$ Coronavirus $\cdot$ SARS-CoV-2 $\cdot$ Leukoencephalopathy $\cdot$ Demyelination $\cdot$ Vasculitis $\cdot$ MRI

\begin{abstract}
Abbreviations
COVID-19 Coronavirus disease 2019

SARS-CoV-2 Severe acute respiratory syndrome coronavirus 2
\end{abstract}

\section{Introduction}

A wide spectrum of neurological disease is recognized in COVID-19 from mild to severe presentations (Mao et al. 2020; Xiong et al. 2021). We report two patients with severe respiratory failure and poor neurological status with novel subacute radiological findings that yield insight into the pathogenesis of COVID-19 leukoencephalopathy.

\section{Case series}

Both patients were symptomatic and tested positive for SARS-CoV-2 PCR on nasopharyngeal swabs. Patient 1 had 2 weeks of illness, and patient 2 had 1 week of illness at home. Both required ICU admission and intubation for severe type-1 respiratory failure secondary to COVID-19

Julie Chandra

julie.chandra@nhs.net

1 Department of Neuroradiology, King's College Hospital NHS Foundation Trust, London, United Kingdom

2 Department of Neurology, King's College Hospital NHS Foundation Trust, London, United Kingdom pneumonitis, IV antibiotics and hemodialysis for acute kidney injury. Neither received extra-corporeal membrane oxygenation nor experimental treatment. Table 1 provides a detailed summary of the clinical presentation, laboratory and imaging investigations and treatment regime for both patients.

Patient 1 was a 61-year-old woman with type-2 diabetes and raised inflammatory markers. After 3 weeks, she was extubated with poor neurological status; she was non-verbal and showed little movement. Brain CT on day 18 and MRI on day 24 demonstrated numerous lesions in the deep cerebral, callosal and cerebellar white matter demonstrating diffusion restriction (Fig. 1). Gadolinium was contraindicated due to renal failure.

Lumbar puncture was normal including negative tests for SARS-CoV-2 RNA RT-PCR, oligoclonal bands, JC virus and antibodies to myelin-oligodendrocyte-glycoprotein and aquaporin-4 (Table 1). EEG supported a moderate encephalopathy. An unusual post-viral acute disseminated encephalomyelitis (ADEM) was hypothesized based on the clinicoradiological findings in particular the splenial and middle cerebellar peduncle involvement, despite the normal CSF. She was treated with a 3-day course of IV methylprednisolone and a tapering course of oral prednisolone. There was clinical response, and by day 34 she could sit, but her neurologic status remained highly variable. Contrast-enhanced MRI at day 46 demonstrated incomplete ring and punctate enhancement in the existing lesions and the presence of microbleeds and gliosis (Fig. 2). In view of potentially active disease, she had 5 days of plasma exchange. MRI at 
Table 1 Summary of clinical presentation, investigations and immunological treatment in both cases

\begin{tabular}{|c|c|c|}
\hline & Patient 1 & Patient 2 \\
\hline $\begin{array}{l}\text { Demographics } \\
\text { Social history }\end{array}$ & 61-year-old female & $\begin{array}{l}\text { 62-year-old male } \\
\text { Ex-smoker }\end{array}$ \\
\hline Past medical history & $\begin{array}{l}\text { Type } 2 \text { diabetes } \\
\text { Hypertension }\end{array}$ & $\begin{array}{l}\text { Hypertension } \\
\text { Diverticular disease }\end{array}$ \\
\hline Inflammatory markers on admission & $\begin{array}{l}\text { WCC } 10.2 \\
\text { CRP } 244\end{array}$ & $\begin{array}{l}\text { WCC } 14.8 \\
\text { CRP } 290\end{array}$ \\
\hline Duration between symptom onset and admission & 14 days & 7 days \\
\hline $\begin{array}{l}\text { Timing from admission to intubation due to COVID-19 } \\
\text { pneumonitis }\end{array}$ & 0 days & 2 days \\
\hline Reason for neurological investigation & $\begin{array}{l}\text { Persisting low level of consciousness } \\
\text { after extubation }\end{array}$ & $\begin{array}{l}\text { Bilateral facial twitching (whilst sedated } \\
\text { and intubated) } \\
\text { Low level of consciousness after extuba- } \\
\text { tion }\end{array}$ \\
\hline Timing from admission to initial brain imaging & $\begin{array}{l}\text { CT } 18 \text { days } \\
\text { MRI } 24 \text { days }\end{array}$ & $\begin{array}{l}\text { CT } 30 \text { days } \\
\text { MRI } 77 \text { days }\end{array}$ \\
\hline Timing of subsequent brain imaging from admission & $\begin{array}{l}\text { MRI day } 46 \text { (post-IVMP) } \\
\text { MRI day } 73 \text { (post-PLEX) }\end{array}$ & MRI day 98 (post-IVMP) \\
\hline Immunological treatment & $\begin{array}{l}\text { Day } 26 \text { IVMP } \times 3 \text { (oral taper) } \\
\text { Day } 62 \text { PLEX } \times 5 \text { days }\end{array}$ & $\begin{array}{l}\text { Day } 50 \text { IVMP } \times 5 \text { (IV taper over } 15 \text { days }) \\
\text { Day } 98 \text { IVMP } \times 3 \\
\text { Day } 98 \text { PLEX } \times 5\end{array}$ \\
\hline Serum immunological markers & $\begin{array}{l}\text { SPE: negative } \\
\text { ANCA: negative } \\
\text { dsDNA: negative } \\
\text { Autoantibody screen: Negative } \\
\text { Anti-GBM: Negative } \\
\text { MOG: negative } \\
\text { AQ4: negative }\end{array}$ & - \\
\hline CSF Markers & $\begin{array}{l}\text { WCC }<5(/ \mathrm{cmm}) \\
\text { RBC }<5(/ \mathrm{cmm}) \\
\text { Protein } 0.36(0.25-0.45 \mathrm{~g} / \mathrm{L}) \\
\text { Glucose } 8.4(\mathrm{mmol} / \mathrm{L}) \\
\text { - }>\text { Serum } 21.1(\mathrm{mmol} / \mathrm{L}) \\
\text { OCB (paired): negative } \\
\text { COVID-19 RNA: negative } \\
\text { Virology: negative } \\
\text { JC virus: negative } \\
\text { Cytology: negative }\end{array}$ & $\begin{array}{l}\text { WCC }<5(/ \mathrm{cmm}) \\
\text { RBC } 8(/ \mathrm{cmm}) \\
\text { Protein } 0.39(0.25-0.45 \mathrm{~g} / \mathrm{L}) \\
\text { Glucose } 5.4(\mathrm{mmol} / \mathrm{L}) \\
\text { - > Serum } 14.7(\mathrm{mmol} / \mathrm{L}) \\
\text { OCB (paired): negative } \\
\text { COVID-19 RNA: negative } \\
\text { Virology: negative } \\
\text { AFBs: negative }\end{array}$ \\
\hline $\begin{array}{l}\text { EEG } \\
\text { (Off sedation) }\end{array}$ & $\begin{array}{l}\text { Day } 24 \\
\text { Moderate encephalopathy }\end{array}$ & $\begin{array}{l}\text { Day } 93 \\
\text { Moderate-severe encephalopathy }\end{array}$ \\
\hline
\end{tabular}

day 73 showed resolution of contrast enhancement with no new lesions (not shown). She improved, and by day 172 she could stand and complete some activities of daily living.

Patient 2 was a 62-year-old male ex-smoker. His admission CRP was high, highlighting a pro-inflammatory phenotype. He had poor neurological status on extubation and remained intubated. Brain CT on day 30 demonstrated widespread cerebral and callosal white matter hypoattenuation with swelling and a cerebellar white matter lesion topographically reminiscent of patient 1 (Fig. 3). He received 5 days of IV methylprednisolone for a co-existent organizing pneumonia and a tapering course of oral prednisolone.

On day 43, he developed intermittent facial twitching but remained intubated. Brain MRI was performed on day
77 , delayed due to his significant cardiorespiratory instability and performed without contrast due to renal failure. This confirmed extensive leukoencephalopathy with deep cerebral, callosal and cerebellar white matter lesions, larger lesions demonstrated ring-shaped diffusion restriction (Fig. 3). EEG off sedation showed moderate-severe encephalopathy. He failed to improve neurologically, and repeat MRI on day 98 showed ongoing diffusion restriction with low apparent diffusion coefficient values (ADC) within the same lesions and microbleeds (Fig. 3). He had spontaneous eye opening but no other movements. Lumbar puncture was normal including negative SARS-CoV-2 RNA RT-PCR (Table 1). He was treated with another 3 days of IV methylprednisolone and 5 days of plasma exchange 


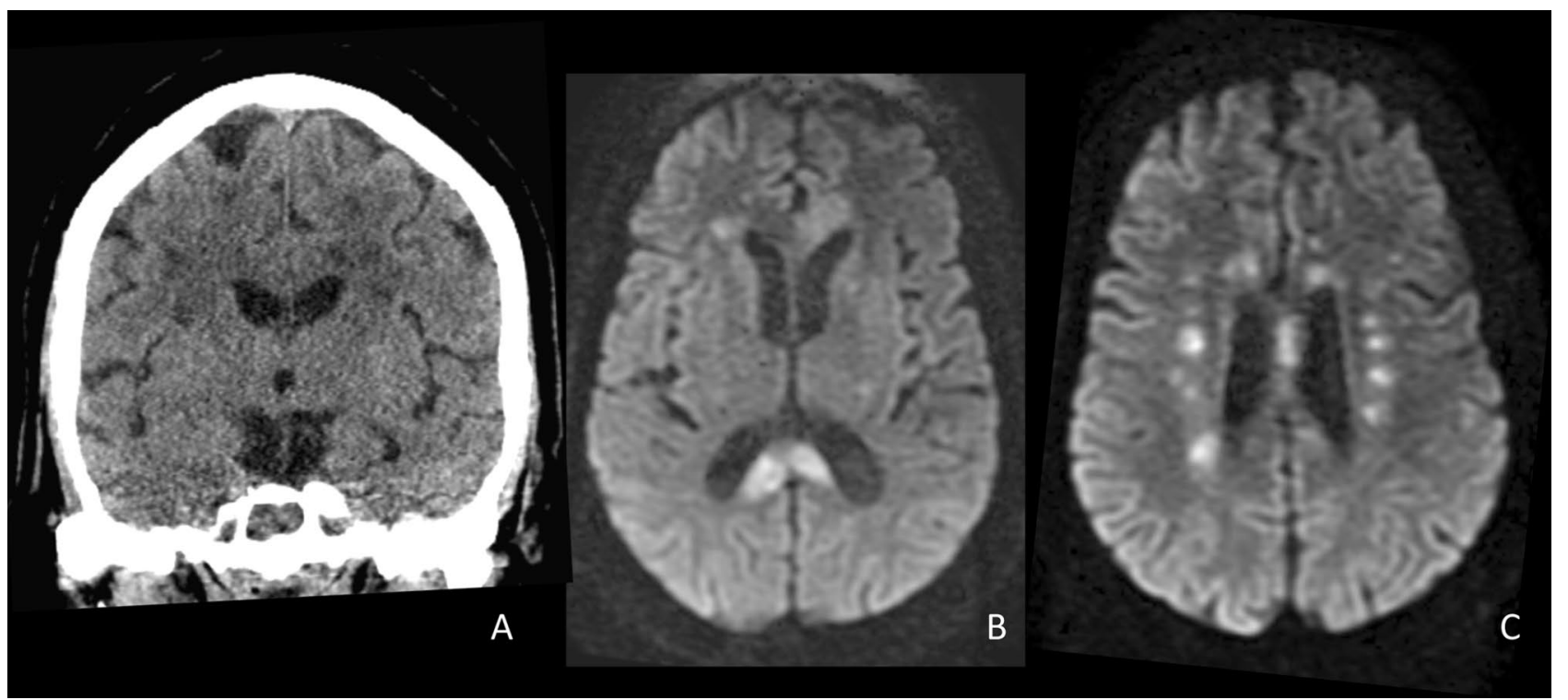

Fig. 1 Patient 1. Coronal brain CT scan (A) shows numerous white matter hypodensities. Brain MRI demonstrates diffusion restriction within many of the lesions involving the corpus callosum (B) and

without improvement. As the prospect of meaningful levels of recovery was not possible, he was converted to a palliative management plan.

\section{Discussion}

What has become clear to clinicians around the world is that SARS-CoV-2 is a disease unlike any other. Acute leukoencephalopathy has been described; however, there are few reports on its imaging evolution (Agarwal et al. 2020b; Lang et al. 2020; Radmanesh et al. 2020b). This is of clinical importance as leukoencephalopathy and cerebral microbleeds are associated with critical illness and higher mortality.

We report incomplete-ring enhancement in our first patient, which could suggest ADEM where breakdown of the blood-brain-barrier facing the white matter occurs at the leading edge of demyelination. However, incomplete-ring enhancement is also common in primary cerebral angiitis, and vasculitis was also considered a differential in our first case (Boulouis et al. 2017). A case report of an ADEM-like illness following mild SARS-CoV-2 infection with typical brain, optic nerve and cord lesions demonstrated an incomplete-ring enhancing brain lesion, but no microbleeds or diffusivity changes were described. In addition, the clinical presentation was in contrast to our critically ill patient with poor neurological status; the latter is the emerging phenotype of COVID-19 leukoencephalopathy (Novi et al. 2020). Of note, reports in the COVID-19 pandemic of other parainfectious immune-mediated neurological conditions such as cytotoxic lesions of the corpus callosum and Guillain-Barré deep cerebral hemispheric white matter $(\mathbf{C})$. The largest lesions in the splenium display a ring-like pattern (B, white arrows). Small cerebellar white matter and middle cerebellar peduncle lesions are not shown

syndrome remain relatively sparse considering the worldwide prevalence (Xiong et al. 2021). However, that does not discount ADEM as a possibility in our case where a clinical response was seen following immunotherapy.

Microbleeds and diffusion restriction are hallmarks of COVID-19 leukoencephalopathy seen in both of our cases, and small-vessel vasculitis has been postulated as the cause of such changes (Agarwal et al. 2020a; Lang et al. 2020). In our second patient, we report novel diffusion restriction with low ADC values persisting over 3 months into admission. This exceeds the expected temporal evolution for diffusion restriction in infarcts or demyelination, which usually normalizes on follow-up. The persistent diffusion restriction in this case of COVID-19 leukoencephalopathy could be secondary to a prolonged ischemic insult in the context of vessel wall inflammation, a phenomenon seen specifically in vasculitis and not occlusive or embolic infarcts (Stanley et al. 2017). However, inflammatory etiologies such as ADEM can rarely show ongoing diffusion restriction. The lack of clinical response to immunotherapy in our second patient could lend support to a dominant vascular etiology although ultimately the high burden, and severity of lesions may be responsible for his poor outcome.

Leptomeningeal enhancement is a common COVID19 neuroimaging finding (Kremer et al. 2020). Whilst the etiology is currently unknown in COVID-19, it is a wellrecognized feature of cerebral vasculitis, not just meningitis (Boulouis et al. 2017). Arterial wall enhancement and thickening have also recently been described in COVID-19 lending support to a vasculitis (Lersy et al. 2020). Vessel wall or angiographic imaging was not possible in our cases due to renal failure and patient instability. The significant 
Fig. 2 Follow-up MRI. The axial (A) and coronal (B) T1-weighted images post-gadolinium demonstrate incompletering contrast enhancement around 2 of the larger callosal and periventricular lesions (white arrowheads) as well as numerous foci of punctate contrast enhancement which numbered 18 in total. A followup scan depicts maturation with gliosis on T2-weighted images (C). Susceptibility-weighted imaging shows punctate microbleeds centred on the T2 lesions (D) most extensive in the splenium of the corpus callosum (white arrows). Isodiffusivity was seen on ADC maps with mild $\mathrm{T} 2$ shine-through on diffusion-weighted images (not shown)

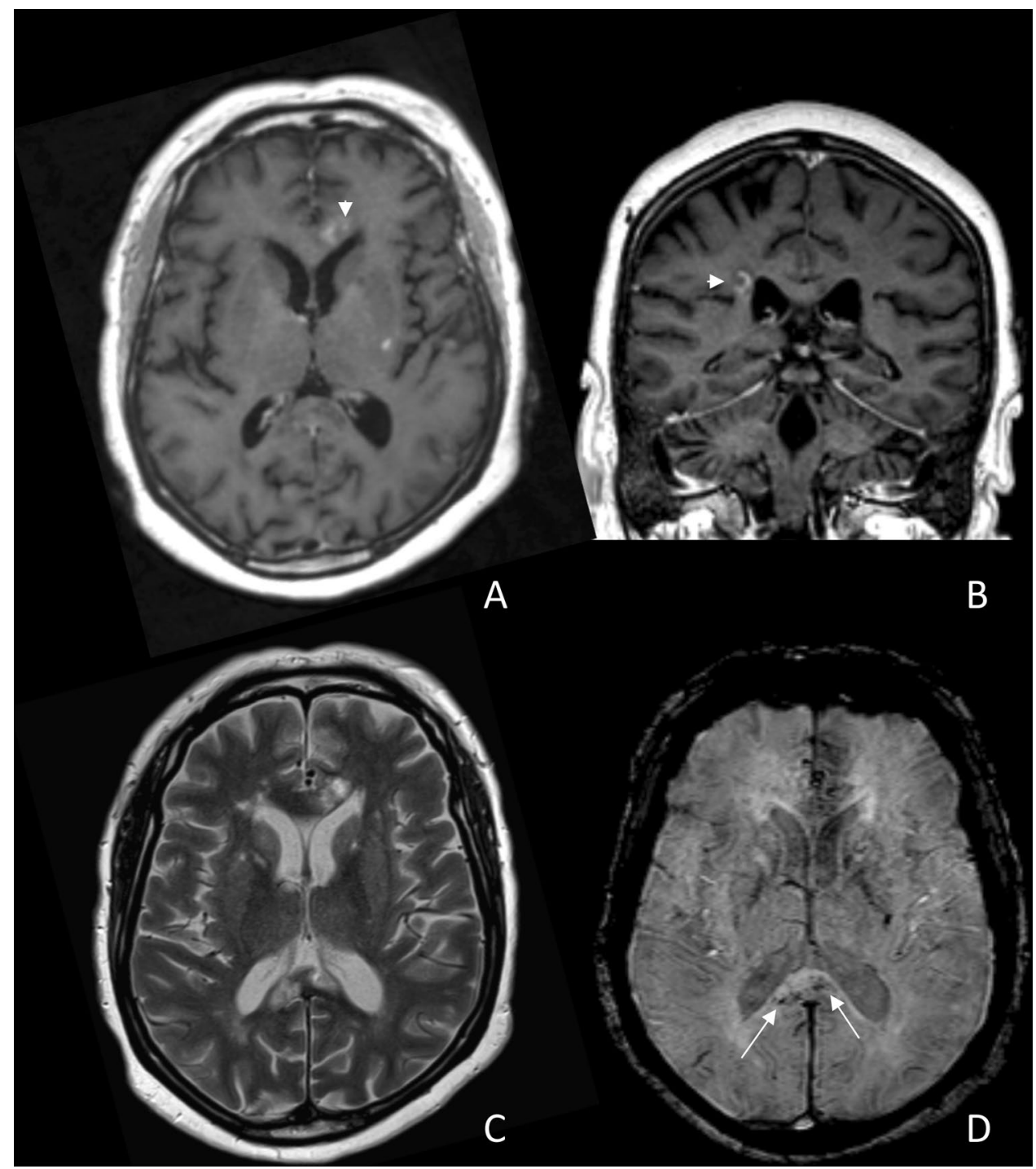

callosal involvement in both our cases goes against embolic infarcts, small vessel disease or a hypoxic-ischemic etiology.

Both our cases depicted lesions showing ring-shaped diffusion restriction, previously described in two cases (Toledano-Massiah et al. 2020). This feature is reminiscent of cytotoxic edema at the leading edge of demyelination. Post-hypoxic necrotizing leukoencephalopathy has been postulated in some COVID-19 cases where there is confluent white matter signal change and diffusion restriction, and in this condition, it is thought to reflect widespread demyelination secondary to ischemia (Radmanesh et al. 2020a). A recent neuropathological study of COVID-19 described vascular and ADEM-like findings and has raised demyelination as secondary to an ischemic insult rather than a primary phenomenon (Reichard et al. 2020).

There is growing evidence for SARS-CoV-2 neuroinvasion, and recently, infection has been shown directly within infarcts in human post-mortem brain studies, within in vitro human brain organoids and in vivo mice models associated with neuronal death and remodeling of the vasculature (Song et al. 2021). The absence of SARS-CoV-2 in the CSF in our cases and the literature does not imply a lack of neuroinvasion but could be due to low PCR sensitivity or CSF penetrance. The constellation of neuroimaging findings in our cases could hypothetically represent the sequelae of SARS-CoV-2 neuroinvasion, virally mediated small-vessel vasculitis and subsequent ischemic demyelination with a leading spreading edge (ring-shaped diffusion restriction, incomplete-ring enhancement) and prolonged ischemia (persistent diffusion restriction).

The clinical picture in critically ill patients remains complex confounded by profound hypoxia and a cytokine storm, and the contribution of thrombotic microangiopathy cannot be discounted. Serial neuroimaging with MRI in critically ill patients with COVID-19 pneumonitis remains clinically challenging. 


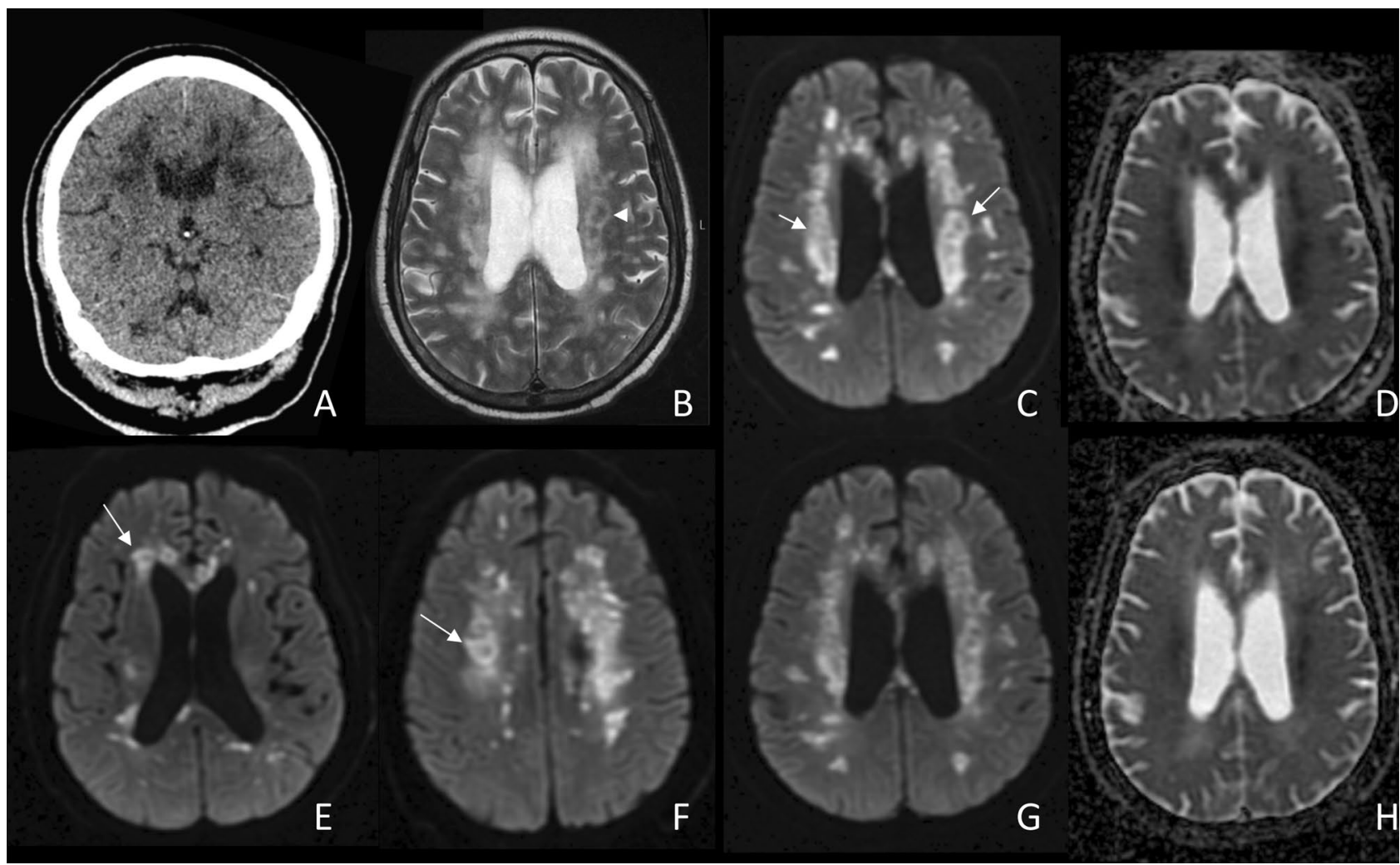

Fig. 3 Patient 2. Coronal CT image (A) shows extensive cerebral white matter hypodensities and a right cerebellar white matter lesion. Delayed brain MRI (B) shows extensive deep watershed cerebral white matter and callosal lesions on $\mathrm{T} 2$-weighted images; larger lesions show ring-like peripheral high $\mathrm{T} 2$ signal with central mild low to isointense signal (white arrowhead). On diffusion-weighted imaging $(\mathbf{C}, \mathbf{E}, \mathbf{F})$, many larger lesions display corresponding ring-

\section{Conclusion}

The constellation of neuroimaging findings in our two cases of COVID-19 leukoencephalopathy and response to immunotherapy in one case supports either vasculitic or demyelinating immune-mediated processes; however, in light of recent evidence of SARS-CoV-2 neuroinvasion and vascular damage, we hypothesize that there is in fact a virally induced potent small-vessel vasculitis and possible ischemic demyelination. Our report aims to help clinicians recognize COVID-19 leukoencephalopathy, the first step in understanding this unique entity and developing therapeutic strategies.

Acknowledgements We thank patient 1 and the next of kin of patient 2 who both provided written informed consent for these case presentations to be published.

\section{Declarations}

Conflict of interest The authors declare no competing interests. like diffusion restriction (white arrows); low values on the apparent diffusion coefficient (ADC) map (D) confirm diffusion restriction. Follow-up MRI shows no new lesions but ongoing diffusion restriction in the same lesions $(\mathbf{G})$ although slightly reduced compared to the initial scan; (H) ADC map correlates. Subtle microhemorrhage is evident in some periventricular lesions on gradient-echo $\mathrm{T} 2 *$ images (not shown)

\section{References}

Agarwal S, Conway J, Nguyen V et al (2020a) Serial imaging of virus-associated necrotizing disseminated acute leukoencephalopathy (VANDAL) in COVID-19. Am J Neuroradiol. https:// doi.org/10.3174/ajnr.a6898

Agarwal S, Jain R, Dogra S et al (2020b) Cerebral microbleeds and leukoencephalopathy in critically ill patients with COVID-19. Stroke. https://doi.org/10.1161/STROKEAHA.120.030940

Boulouis G, De Boysson H, Zuber M et al (2017) Primary angiitis of the central nervous system: magnetic resonance imaging spectrum of parenchymal, meningeal, and vascular lesions at baseline. Stroke. https://doi.org/10.1161/STROKEAHA.116.016194

Kremer S, Lersy F, Anheim M et al (2020) Neurologic and neuroimaging findings in patients with COVID-19: a retrospective multicenter study. Neurology. https://doi.org/10.1212/WNL. 0000000000010112

Lang M, Buch K, Li MD et al (2020) Leukoencephalopathy associated with severe COVID-19 infection: sequela of hypoxemia? AJNR Am J Neuroradiol. https://doi.org/10.3174/ajnr.A6671

Lersy F, Anheim M, Willaume T et al (2020) Cerebral vasculitis of medium-sized vessels as a possible mechanism of brain damage in COVID-19 patients. J Neuroradiol. https://doi.org/10.1016/j. neurad.2020.11.004 
Mao L, Jin H, Wang M et al (2020) Neurologic manifestations of hospitalized patients with coronavirus disease 2019 in Wuhan, China. JAMA Neurol. https://doi.org/10.1001/jamaneurol.2020.1127

Novi G, Rossi T, Pedemonte E et al (2020) Acute disseminated encephalomyelitis after SARS-CoV-2 infection. Neurol Neuroimmunol Neuroinflammation. https://doi.org/10.1212/NXI.0000000000000797

Radmanesh A, Derman A, Ishida K (2020a) COVID-19-associated delayed posthypoxic necrotizing leukoencephalopathy. J Neurol Sci

Radmanesh A, Derman A, Lui YW et al (2020) COVID-19-associated diffuse leukoencephalopathy and microhemorrhages. Radiology. https://doi.org/10.1148/radiol.2020202040

Reichard RR, Kashani KB, Boire NA et al (2020) Neuropathology of COVID-19: a spectrum of vascular and acute disseminated encephalomyelitis (ADEM)-like pathology. Acta Neuropathol. https://doi.org/10.1007/s00401-020-02166-2

Song E, Zhang C, Israelow B et al (2021) Neuroinvasion of SARSCoV-2 in human and mouse brain. J Exp Med. https://doi.org/10. 1084/jem.20202135
Stanley E, Murphy S, Kavanagh E (2017) Letter by Stanley et al Regarding article, "primary angiitis of the central nervous system magnetic resonance imaging spectrum of parenchymal, meningeal, and vascular lesions at baseline." Stroke

Toledano-Massiah S, Badat N, Leberre A et al (2020) Unusual brain MRI pattern in 2 patients with COVID-19 acute respiratory distress syndrome. Am J Neuroradiol. https://doi.org/10.3174/ajnr. a6817

Xiong W, Kwan P, Zhou D et al (2021) Acute and late neurological complications of COVID19: the quest for evidence. Brain. https:// doi.org/10.1093/brain/awaa294

Publisher's Note Springer Nature remains neutral with regard to jurisdictional claims in published maps and institutional affiliations. 